\title{
Treatment compliance and severe adverse events limit the use of tyrosine kinase inhibitors in refractory thyroid cancer
}

This article was published in the following Dove Press journal:

OncoTargets and Therapy

3 September 2015

Number of times this article has been viewed

\section{Alexandra Chrisoulidou \\ Stylianos Mandanas \\ Efterpi Margaritidou \\ Lemonia Mathiopoulou \\ Maria Boudina \\ Konstantinos Georgopoulos \\ Kalliopi Pazaitou- \\ Panayiotou}

Department of Endocrinology,

Theagenio Cancer Hospital,

Thessaloniki, Greece
Correspondence: Kalliopi PazaitouPanayiotou

Department of Endocrinology, Theagenio Cancer Hospital, 2 Al Simeonidi Street,

Thessaloniki 54007, Greece

$\mathrm{Tel}+302310898618$

Fax +302310898809

Email kpazaitoupanayiotou@gmail.com
Objective: The aim of the present study was to assess patient compliance with tyrosine kinase inhibitor (TKI) treatment used for refractory and progressive thyroid cancer, in addition to the efficacy and serious adverse events associated with these agents.

Methods: We retrospectively analyzed data from adult patients with metastatic differentiated or medullary thyroid cancer unresponsive to conventional treatment and treated with TKIs. Patients received treatment until disease progression or onset of serious adverse events, or until they expressed an intention to stop treatment.

Results: Twenty-four patients received TKIs. The median duration of treatment was four (range: 1-19) cycles. The most frequent adverse events were fatigue, nausea, diarrhea, hypertension, and stomatitis, and the most severe were nasal bleeding, diarrhea, heart failure, rhabdomyolysis, renal failure, QT prolongation, neutropenia, and severe fatigue. Dose reduction was required in eight patients, while five decided to terminate TKI therapy because adverse events impaired their everyday activities. During therapy, two patients showed a partial response and three showed stable disease. The lungs were the metastatic sites favoring a response to treatment.

Conclusion: Patient selection and meticulous pretreatment education are necessary in order to ensure adherence with TKI therapy. If adverse events appear, dose reduction or temporary treatment interruption may be offered because some adverse events resolve with continuation of treatment. In the event of serious adverse events, treatment discontinuation is necessary.

Keywords: medullary thyroid carcinoma, differentiated thyroid cancer, TKIs, sorafenib, sunitinib, vandetanib

\section{Introduction}

Differentiated thyroid cancer (DTC) is the most frequent type of thyroid cancer, ${ }^{1}$ comprising more than $90 \%$ of all cases. Thyroidectomy and radioactive iodine (RAI) ablation remain the cornerstones of treatment. Although the disease course is indolent in the majority of patients, aggressive cases with metastases (refractory to conventional treatment) exist, and remain challenging for clinicians. Over recent decades, the trend of an increasing incidence of thyroid cancer has inevitably given rise to a number of patients who present with aggressive disease and eventually succumb to it. ${ }^{2}$ The survival rate in patients with RAI-refractory metastatic DTC has been estimated to be $10 \%$ at 10 years. ${ }^{3}$ Until recently, the therapeutic options available for patients with progressive, metastatic, RAI-refractory DTC have been limited.

C-cell-derived medullary thyroid carcinoma (MTC), although uncommon, has a much worse prognosis than iodine-positive DTC, which is approximately the same with RAI-refractory DTC. ${ }^{4}$ In most cases, MTC is already metastatic at initial 
presentation, with no available effective therapeutic options other than surgery, when possible.

Novel tyrosine kinase inhibitors (TKIs), such as sorafenib, sunitinib, cabozantinib, and vandetanib, have been used recently for the treatment of refractory cases of thyroid cancer ${ }^{5-7}$ where all conventional treatment options (surgery, RAI, chemotherapy) have been proven ineffective. These molecules inhibit cellular signaling by targeting multiple tyrosine kinase receptors as well as platelet-derived growth factor receptors and vascular endothelial growth factor receptors, which play a role in both tumor angiogenesis and proliferation of tumor cells. Simultaneous inhibition of these targets leads to reduced tumor vascularization, apoptosis of cancer cells, and ultimately tumor shrinkage. Some Phase II and III trials ${ }^{8,9}$ have reported promising results regarding favorable response rates in metastatic thyroid cancer that has been non-responsive to conventional treatment. Recently, vandetanib and cabozantinib were approved for patients with MTC and sorafenib was approved for those with DTC. ${ }^{910}$ However, because both agents target many different receptors, they have numerous side effects, including hematological, skin, and cardiac toxicities that may have a negative impact on patients' quality of life.

The aim of the present study was to assess the effects of TKI inhibitors in a cohort of patients with refractory and progressive thyroid cancer (DTC or MTC), in particular severe adverse events during TKI therapy, ability and willingness of patients to remain on long-term treatment, and response rates.

\section{Materials and methods}

Data from patients with metastatic refractory thyroid cancer who received TKIs from April 2009 to December 2014 were retrospectively analyzed. Approval for the drug administration to every patient was obtained from the insitutional scientific committee and the National Drug Organization. Each patient was informed in detail about the efficacy and possible adverse events (minor or major) associated with the treatment. The same doctor (SM) educated all patients and confirmed their understanding of the possible complications of therapy and its expected efficacy. All patients agreed to start TKI therapy after they had been fully informed and given their written consent.

All patients were of Caucasian origin, of similar socioeconomic status, and lived in Northern Greece (population approximately 3 million inhabitants). Initially, candidates for sunitinib were those who had metastatic DTC to the lungs or bones refractory to iodine treatment and progressive disease within 12 months before baseline, documented by computed tomography (CT) or magnetic resonance imaging (MRI) and thyroglobulin; and for sorafenib were those who had metastatic MTC to the liver (with unresectable metastatic lesions) or bones and progressive disease within 12 months before baseline, documented by CT or MRI and by an increase in serum calcitonin levels. After approval by the European Medicines Agency (EMA) for sorafenib and vandetanib to be used in the treatment of DTC and MTC, respectively, patients with refractory thyroid cancer who were candidates for TKIs received these drugs. All patients were in general good health, defined as an Eastern Cooperative Oncology Group performance status of 0 or $1 .{ }^{11}$ A clinical evaluation was performed, and normal blood pressure, with appropriate treatment where necessary, was achieved before initiation of TKI treatment. All patients had normal liver, renal, and bone marrow function. No abnormalities were identified on baseline electrocardiography or echocardiography. Baseline QT intervals were in the normal range.

Sorafenib $400 \mathrm{mg}$ was given orally twice daily continuously, sunitinib was given as $50 \mathrm{mg}$ once daily on a 4-2 schedule (4 weeks of treatment followed by 2 -week intervals without therapy), and vandetanib was given as $300 \mathrm{mg}$ once daily. A 4-week treatment with each agent defined a cycle. Patients were evaluated weekly for the first cycle and monthly thereafter, in order to determine well-being and Eastern Cooperative Oncology Group performance status. At each visit, clinical examination, blood pressure, weight, and a complete blood count, urinalysis, and serum biochemical measurements were performed. Cardiac evaluation with an electrocardiogram and/or echocardiogram was periodically performed and when indicated according to patient symptoms. Extensive discussion, evaluation of predisposing factors (such as diabetes), and written information was provided regarding the side effects of the medication. Patients were advised to prevent stomatitis with systematic oral care hygiene using a soft toothbrush and bland rinses, and to use local anesthetics as/if needed. ${ }^{12}$ The patients were also asked to limit exposure of the hands and feet to hot water, chemicals, or sources of heat (eg, sitting in the sun), to take cool showers, wear wellventilated shoes, and apply skin care creams to keep the hands moist in order to prevent and manage hand-foot syndrome. ${ }^{13}$ Patients were instructed to report any new symptom or sign to their physicians at every visit.

Answers to questions regarding changes in daily habits were recorded. Any adverse events were documented and graded using the National Cancer Institute Common Terminology Criteria for Adverse Events, version 3.0. ${ }^{14}$ The presence of adverse events that would need dose reduction or discontinuation of therapy was also documented at every visit. 
Response to therapy was estimated by CT or MRI after three and six cycles and every four cycles thereafter. Disease progression was assessed according to the Response Evaluation Criteria in Solid Tumors (version 1.0) ${ }^{15}$ Thyroidstimulating hormone (TSH) for all patients, thyroglobulin for DTC patients, and calcitonin for MTC patients were also evaluated concomitantly. The study was approved by the institutional review board. The presented data are expressed where appropriate as the median and range.

\section{Results}

Twenty-four patients (14 males, ten females) were identified from our clinical database to have received TKIs since April 2009. Fourteen patients had DTC and ten had MTC. Their median age was 58.29 (range: $42-74$ ) years. The histological characteristics of the tumors are presented in Table 1.

Table I Characteristics of patients who received tyrosine kinase inhibitors

\begin{tabular}{|c|c|}
\hline Characteristics & Patients $(n=24)$ \\
\hline Median age (years) & 58.29 \\
\hline (range) & $(42-74)$ \\
\hline \multicolumn{2}{|l|}{ Sex, n (\%) } \\
\hline Male & $14(58)$ \\
\hline Female & $10(42)$ \\
\hline \multicolumn{2}{|l|}{ Histology of TC (n) } \\
\hline Papillary TC & 6 \\
\hline Follicular TC & 3 \\
\hline Hurthle cell & 4 \\
\hline Poorly differentiated & 1 \\
\hline MTC & 10 \\
\hline \multicolumn{2}{|l|}{ Metastatic disease (n) } \\
\hline Mediastinal mass & 5 \\
\hline Lungs & 14 \\
\hline Liver & 9 \\
\hline Bone & 7 \\
\hline \multicolumn{2}{|l|}{ ECOG performance status, $\mathrm{n}(\%)$} \\
\hline 0 & $22(91)$ \\
\hline 1 & $2(9)$ \\
\hline \multicolumn{2}{|l|}{ Time since diagnosis of TC (months) } \\
\hline DTC & $29(15-108)$ \\
\hline MTC & $64.3(6-108)$ \\
\hline \multicolumn{2}{|l|}{ Previous treatment, $\mathrm{n}$} \\
\hline \multicolumn{2}{|l|}{ DTC } \\
\hline Surgery & 14 \\
\hline Systemic chemotherapy & 3 \\
\hline Radioiodine (median dose, range) & $14(319,150-900 \mathrm{mCi})$ \\
\hline External beam irradiation & 6 \\
\hline \multicolumn{2}{|l|}{ MTC } \\
\hline Surgery & 10 \\
\hline Systemic chemotherapy & 2 \\
\hline Radiofrequency ablation & 2 \\
\hline Chemoembolization & 4 \\
\hline External beam irradiation & 4 \\
\hline
\end{tabular}

Note: Values expressed as the median (range).

Abbreviations: ECOG, Eastern Cooperative Oncology Group; TC, thyroid cancer; DTC, differentiated thyroid cancer; MTC, medullary thyroid carcinoma.
All patients had distant metastases located at one or more sites (liver $37.5 \%$, lung $58 \%$, bone $29.1 \%$, and lower mediastinum $20.8 \%$ ). The time between diagnosis of thyroid carcinoma and treatment with a TKI varied from a minimum of 6 months to a maximum of 9 years. Previous treatments included thyroidectomy (in all patients) with/without lymph node dissection and administration of RAI (in all DTC patients), external beam radiation, and local therapies for liver metastases (radiofrequency ablation and/or chemoembolization; for details, Table 1). All applied treatment was terminated at least 6 months prior to initiation of TKI therapy.

\section{Adverse events}

The duration of TKI treatment ranged from one to 19 cycles. Younger patients tolerated longer treatment (Table 2). The first adverse events appeared after approximately 2 weeks of therapy, and were fatigue, mild diarrhea, and stomatitis. During treatment, the most commonly reported adverse events were diarrhea $(81.25 \%)$, stomatitis $(75 \%)$, fatigue $(62.5 \%)$, and skin adverse effects ( $81.25 \%$, comprising handfoot syndrome, skin discoloration, and rash, Tables 2 and 3). A minority of patients developed anemia, neutropenia, and elevated triglyceride levels (Table 2). Although many adverse events were grade 1 and 2, 15 patients (62.5\%) reported grade 3 and 4 toxicities (Tables 2 and 3). In particular, one female patient developed painful vulvar ulcers and refused to continue treatment with sunitinib even in lower dose, one man developed many ulcers across the surface of the scrotum while taking sorafenib, and there were one case with renal failure and one case with QT prolongation where both were on vandetanib. Two cases in particular warrant mention. The first was a 73-year-old female patient who developed congestive heart failure (ejection fraction $35 \%$ ) while on sunitinib. This severe adverse event led to immediate drug withdrawal, with subsequent improvement of cardiac function. The second case was a man with RAIrefractory thyroid cancer in whom intense muscle pain and muscle weakness suddenly appeared 1 month after initiation of sorafenib, along with a nine-fold increase in creatine phosphokinase.

Reduction of the sunitinib dose from 50 to $37.5 \mathrm{mg}$ once daily was required in five patients receiving sunitinib due to neutropenia (two patients), hypertension (two patients), and intense fatigue (one patient); from 400 to $200 \mathrm{mg}$ twice daily for sorafenib in one patient due to scrotal ulcers; and from 300 to $200 \mathrm{mg}$ for vandetanib in two patients (one due to renal failure and one due to QT prolongation). Sequential therapy was attempted with sunitinib followed by sorafenib in two patients due to severe neutropenia caused by sunitinib. 


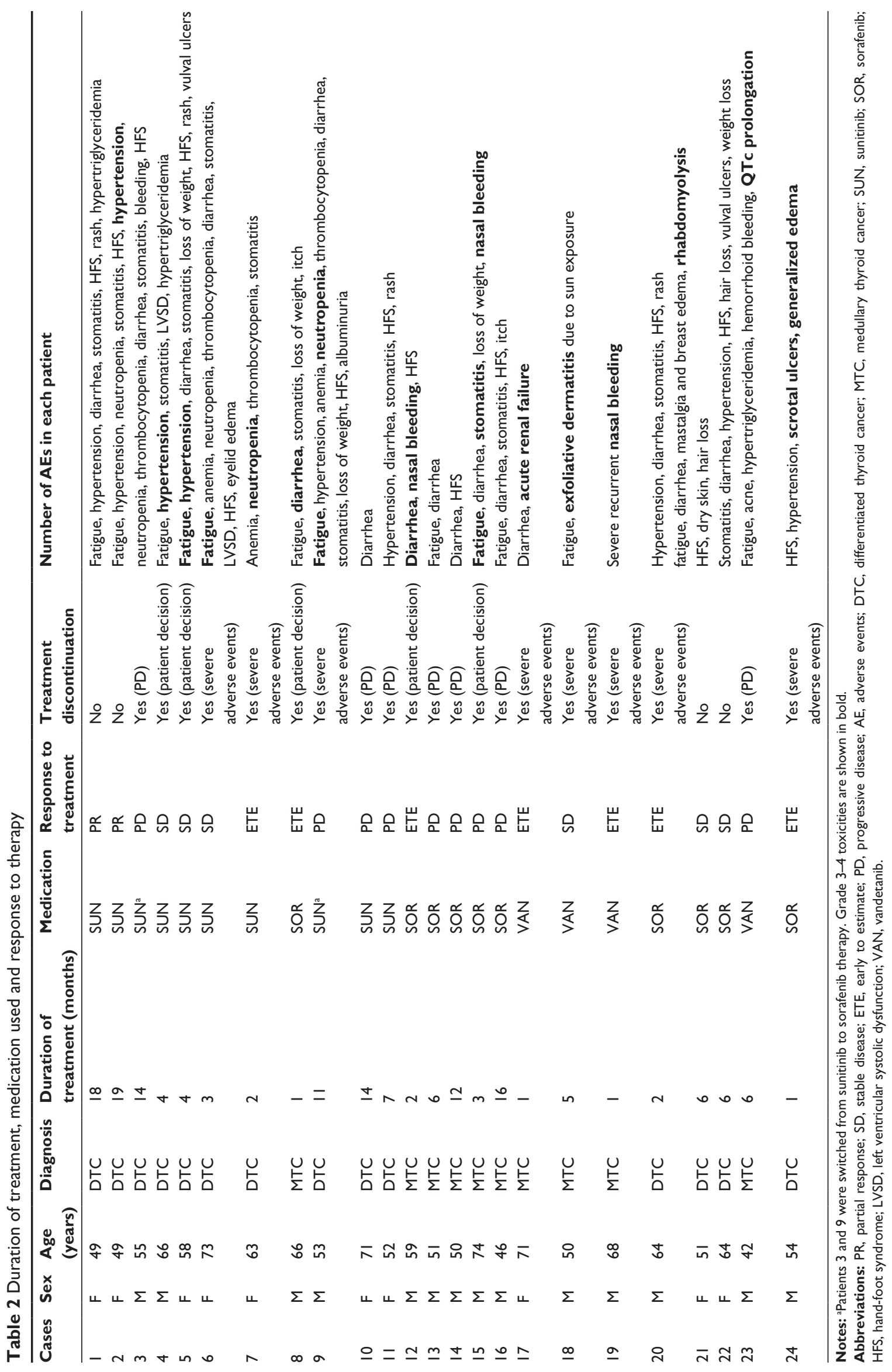


Table 3 Adverse events in patients during treatment with tyrosine kinase inhibitors

\begin{tabular}{|c|c|c|c|}
\hline Adverse event & $\begin{array}{l}\text { Grade I-2 } \\
\text { CTC }\end{array}$ & $\begin{array}{l}\text { Grade } 3 \\
\text { CTC }\end{array}$ & $\begin{array}{l}\text { Grade } 4 \\
\text { CTC }\end{array}$ \\
\hline & $\mathbf{n}$ & $\mathbf{n}$ & $\mathbf{n}$ \\
\hline Fatigue & 9 & 3 & I \\
\hline Stomatitis & 12 & 1 & 0 \\
\hline Loss of weight & 5 & 0 & 0 \\
\hline Diarrhea & 14 & 2 & 0 \\
\hline HFS, skin discoloration & 13 & 0 & 0 \\
\hline Skin reactions (rash, acne) & 5 & 0 & 0 \\
\hline $\begin{array}{l}\text { Exfoliative dermatitis due } \\
\text { to sun exposure }\end{array}$ & 0 & 1 & 0 \\
\hline Scrotal/vulval ulcers & 1 & I & 0 \\
\hline Neutropenia & 3 & I & I \\
\hline Thrombocytopenia & 4 & 0 & 0 \\
\hline Anemia & 3 & 0 & 0 \\
\hline Decreased ejection fraction & I & I & 0 \\
\hline Hypertension & 6 & 3 & 0 \\
\hline Bleeding & 2 & 2 & I \\
\hline Hypertriglyceridemia & 3 & 0 & 0 \\
\hline Renal disease & 0 & I & 0 \\
\hline Rhabdomyolysis & 0 & 1 & 0 \\
\hline Blurred vision & I & 0 & 0 \\
\hline QTc prolongation & 0 & I & 0 \\
\hline
\end{tabular}

Notes: Column 2 shows the number of patients who developed grade 1 and 2 toxicities; columns 3 and 4 shows the number of patients with grade 3 and grade 4 toxicities, respectively.

Abbreviations: CTC, common toxicity criteria; HFS, hand-foot syndrome.

Five patients (20.8\%) opted to stop treatment after $1-4$ cycles, regardless of the severity of the adverse event, because of inability to work long hours, decreased appetite, and restriction of outdoor activities. All adverse events encountered are shown in Table 3.

\section{Overall response}

Overall response, including stable disease and partial response, was estimated for the 17 of the 24 patients who continued treatment for more than three cycles (Table 2). Two patients with DTC showed improvement of lung metastases after 6 months of TKI administration as documented by CT, which improved further as treatment was continued. Metastatic sites favoring response were the lungs. At the time of collecting the data, all patients with DTC were alive; however, six of the ten MTC patients had died. There were no deaths related to TKI therapy.

\section{Tumor markers}

Median TSH was 0.26 (range: $0.01-1.44$ ) $\mu \mathrm{IU} / \mathrm{mL}$ at baseline and $0.48(0.09-3.6) \mu \mathrm{IU} / \mathrm{mL}$ at 3 months. In three patients, an increase in thyroxine dose was decided. Thyroglobulin levels at baseline on thyroxine were $148(1.3-7,572) \mathrm{ng} / \mathrm{mL}$ and at 3 months were $108(1.3-1,629) \mathrm{ng} / \mathrm{mL}$, while the calcitonin levels were 8,107 $(874-8,920) \mathrm{pg} / \mathrm{mL}$ at baseline and 4,901 $(441-5,068) \mathrm{pg} / \mathrm{mL}$ at 3 months.

\section{Discussion}

A high treatment dropout rate was observed in this study, mainly due to drug toxicity along with reluctance of patients to continue treatment. Younger patients tolerated longer treatment, indicating that these patients were perhaps more motivated and willing to receive and continue therapy. The patients presented a high frequency of minor and major adverse events while on treatment. A preferential effect of TKI therapy on metastatic disease to the lung was observed. ${ }^{16}$ Finally, a non-significant trend for reduction of both thyroglobulin and calcitonin serum levels after the use of TKIs was also observed. Because a considerable number of patients (13 of 24) discontinued therapy due to side effects and not disease progression, calculation of progression-free survival or even overall survival would not provide an insight into the efficacy of TKI treatment in this cohort.

With the exception of anaplastic thyroid cancer, patients with MTC and DTC have in most cases a good prognosis, with a 5 -year survival exceeding $60 \%$ for $\mathrm{MTC}^{17}$ and $90 \%$ for DTC. ${ }^{18}$ In this study, the survival of MTC cases was shorter than for DTC patients. Furthermore, while the disease is progressing, patients usually maintain a good quality of life. As a result, they have the expectation that applied therapies would not interfere with their normal everyday life and activities.

In the present study, we used sorafenib for the treatment of MTC patients (when vandetanib was not approved for the treatment of MTC) based on data concerning its activity at the RET and vascular endothelial growth factor receptors ${ }^{19}$ and from two studies published at that time showing a response in $40 \%$ of patients ${ }^{20}$ and stable disease in $50 \%$ of MTC patients. ${ }^{8}$ After approval of vandetanib, patients with MTC received this drug. On the other hand, sunitinib, known as a multitargeted inhibitor at that time, ${ }^{21}$ was administered to RAI-refractory DTC patients in an open-label Phase II study and showed a $13 \%$ partial response rate and a $68 \%$ disease stabilization rate. ${ }^{22}$ Very recently, data have been presented from DECISION, a randomized, double-blind Phase III trial examining the efficacy and safety of sorafenib versus placebo in RAI-refractory DTC patients. ${ }^{23}$ Four hundred and seventeen patients from 77 centers in the USA and Europe were randomized to receive either sorafenib or placebo. The study indicated that sorafenib improved progression-free survival by 5 months over placebo. In another randomized, double-blind Phase III study that included 331 patients with 
MTC, 231 patients received vandetanib and 100 received placebo. In that study, prolongation of progression-free survival was observed in patients who received vandetanib when compared with those who received placebo. ${ }^{10}$ A direct comparison with the earlier findings cannot be made because the design of our study did not include a placebo group or calculation of progression-free survival.

Looking at the efficacy and adverse events between DTC and MTC patients, we can conclude that: sunitinib or sorafenib therapy in DTC patients was more efficacious than sorafenib or vandetanib in MTC patients, given that partial response and stable disease was seen only in DTC patients and adverse events were similar between DTC and MTC patients (Table 2).

Cardiac and hematological side effects were the most clinically important adverse events presented during therapy, and were the most significant medical reasons for withdrawing treatment. In general, cardiac toxicities, from changes on the electrocardiogram and left ventricular ejection fraction abnormalities to severe congestive heart failure and acute coronary syndromes, should be suspected and monitored for during TKI therapy. ${ }^{24,25}$ In our cohort, hematological toxicity of all grades was seen in five of our 24 patients and grade 3-4 events occurred in two patients. Epistaxis, seen in three patients, was an additional reason to withdraw treatment. These results are comparable with the published data for all-grade hematological toxicity of $60 \%-70 \%$ and grade $3-4$ toxicity of $6 \%-8 \% .{ }^{26}$ In addition, we observed hand-foot syndrome in $58.33 \%$ of patients, which is similar to published data reporting rates of $60 \%-91 \% .{ }^{27}$ These skin reactions tend to appear early on in treatment and improve after the first six cycles, ${ }^{28}$ and were also seen in the present study. Finally, fatigue, which probably represents a multifactorial toxicity during use of TKIs, ${ }^{29}$ is debilitating and at times difficult to overcome, as suggested by our findings.

Grade 3 and/or 4 toxicities are an indication for treatment discontinuation; however, lower-grade side effects may also lead to treatment withdrawal, especially when they appear in clusters and persist for a long time. Apart from implementation of supportive measures, ${ }^{30}$ as cited in our methodology, we applied drug discontinuation and dose reduction to alleviate symptoms in patients with toxicities. ${ }^{23}$ Recently, a schedule change from 4 weeks on/2 weeks off during treatment with sunitinib to 2 weeks on/ 1 week off has resulted in significantly fewer events of hand-foot syndrome and fatigue. ${ }^{31}$ This alternative dosing led to fewer grade 3/4 adverse events without compromising drug efficacy, and so far appears superior to dose reduction. ${ }^{32}$ Adverse events with one TKI agent do not preclude the use of another drug in this category; 33 improvement of hematological toxicity was observed in two patients when treatment was switched from sunitinib to sorafenib.

Previous reports advocate the use of tumor markers (thyroglobulin and calcitonin) as surrogate markers ${ }^{34}$ for follow-up in treated patients, along with CT and MRI. Although our patient population was small, we also observed a non-significant trend for reduction of tumor markers with treatment. An elevation in TSH was observed, and thyroxine was increased in three patients. In athyreotic patients, increases in TSH with TKI treatment have been previously documented in $17 \%-33 \%$ of patients, depending on the drug used. ${ }^{30}$ TKIs induce hypothyroidism, probably associated with poor absorption of thyroxine along the gastrointestinal tract as a consequence of diarrhea, thyroid hormone metabolism, or reduction of TSH clearance. ${ }^{35}$

This was a retrospective study and included a relatively small number of patients. However, the fact that these data reflect the experience of a single center using standard therapeutic criteria and treating patients from a specific region could counterbalance some of the limitations. Further, since data related to specific populations are not widely available, the present series adds valuable information related to treatment with TKIs. Small groups of patients are common in this research area, as seen in other recently published studies that refer to similar numbers of patients ${ }^{36-38}$ and contain mixed DTC and MTC populations. ${ }^{16,39,40}$

\section{Conclusion}

TKIs represent a valuable therapeutic option in patients with thyroid cancer. However, these agents should be used with caution and under expert supervision because of their adverse events. Younger patients tolerate treatment better. In patients who continue therapy, adverse events may improve over time and become more tolerable. Apart from thorough education of selected patients, topical therapies, dose reduction of the administered TKI, temporary treatment interruption, and/or permanent discontinuation in severe cases may be considered in those not able to continue long-term treatment. As toxicities impact negatively on patients' quality of life and often restrict their everyday activities, candidate patients must be meticulous selected and close follow-up should be applied.

\section{Disclosure}

The authors report no conflicts of interest in this work. The authors alone are responsible for the content and writing of the paper. 


\section{References}

1. Ito Y, Nikiforov YE, Schlumberger M, Vigneri R. Increasing incidence of thyroid cancer: controversies explored. Nat Rev Endocrinol. 2013;9: 178-184.

2. Kilfoy BA, Zheng T, Holford TR, et al. International patterns and trends in thyroid cancer incidence, 1973-2002. Cancer Causes Control. 2009; 20:525-531.

3. Durante C, Haddy N, Baudin E, et al. Long-term outcome of 444 patients with distant metastases from papillary and follicular thyroid carcinoma: benefits and limits of radioiodine therapy. J Clin Endocrinol Metab. 2006:91:2892-2899.

4. American Thyroid Association Guidelines Task Force, Kloos RT, Eng C, Evans DB, et al. Medullary thyroid cancer: management guidelines of the American Thyroid Association. Thyroid. 2009;19:565-612.

5. Kloos RT, Ringel MD, Knopp MV, et al. Phase II trial of sorafenib in metastatic thyroid cancer. J Clin Oncol. 2009;27:1675-1684.

6. Klein Hesselink EN, Steenvoorden D, Kapiteijn E, et al. Therapy of endocrine disease. Response and toxicity of small molecule tyrosine kinase inhibitors in patients with thyroid carcinoma: a systematic analysis. Eur J Endocrinol. 2015;172:R215-R225.

7. Cabanillas ME, Brose MS, Holland J, Ferguson KC, Sherman SI. A phase I study of cabozantinib (XL184) in patients with differentiated thyroid cancer. Thyroid. 2014;24:1508-1514.

8. Lam ET, Ringel MD, Kloos RT, et al. Phase II clinical trial of sorafenib in metastatic medullary thyroid cancer. J Clin Oncol. 2010;28: 2323-2330.

9. Elisei R, Schlumberger MJ, Müller SP, et al. Cabozantinib in progressive medullary thyroid cancer. J Clin Oncol. 2013;31:3639-3646.

10. Wells SA Jr, Robinson BG, Gagel RF, et al. Vandetanib in patients with locally advanced or metastatic medullary thyroid cancer: a randomized, double-blind phase III trial. J Clin Oncol. 2012;30:134-141.

11. Oken MM, Creech RH, Tormey DC, et al. Toxicity and response criteria of the Eastern Cooperative Oncology Group. Am J Clin Oncol. 1982;5: 649-655

12. Keefe DM, Schubert MM, Elting LS, et al; Mucositis Study Section of the Multinational Association of Supportive Care in Cancer and the International Society for Oral Oncology. Updated clinical practice guidelines for the prevention and treatment of mucositis. Cancer. 2007;109:820-831.

13. Manchen E, Robert C, Porta C. Management of tyrosine kinase inhibitor-induced hand-foot skin reaction: viewpoints from the medical oncologist, dermatologist, and oncology nurse. J Support Oncol. 2011; 9:13-23.

14. US Department of Health and Human Services. Common terminology criteria for adverse events (CTCAE). In: Protocol development. US National Institutes of Health, 2010. Available from: http://ctep.cancer. gov/protocolDevelopment. Accessed July 24, 2015.

15. Therasse P, Arbuck SG, Eisenhauer EA, et al. New guidelines to evaluate the response to treatment in solid tumors. European Organization for Research and Treatment of Cancer, National Cancer Institute of the United States, National Cancer Institute of Canada. J Natl Cancer Inst. 2000;92:205-216.

16. Capdevila J, Iglesias L, Halperin I, et al. Sorafenib in metastatic thyroid cancer. Endocr Relat Cancer. 2012;19:209-216.

17. Abraham DT, Low TH, Messina M, et al. Medullary thyroid carcinoma: long-term outcomes of surgical treatment. Ann Surg Oncol. 2011;18: 219-225.

18. Davies L, Welch HG. Increasing incidence of thyroid cancer in the United States, 1973-2002. JAMA. 2006;295:2164-2167.

19. Ball DW. Medullary thyroid cancer: therapeutic targets and molecular markers. Curr Opin Oncol. 2007;19:18-23.

20. Kober F, Hermann M, Handler A, Krotla G. Effect of sorafenib in symptomatic metastatic medullary thyroid cancer. J Clin Oncol. 2007 25 Suppl 18:S14065.

21. Faivre S, Delbaldo C, Vera K, et al. Safety, pharmacokinetics, and antitumor activity of SU11248, a novel oral multitarget tyrosine kinase inhibitor, in patients with cancer. J Clin Oncol. 2006;24:25-35.
22. Ravaud A, de la Fouchardiere C, Courbon F, et al. Sunitinib in patients with refractory advanced thyroid cancer: the THYSU phase II trial. J Clin Oncol. 2008;26 Suppl 15:S6058.

23. Brose MS, Nutting CM, Jarzab B, et al; DECISION investigators. Sorafenib in radioactive iodine-refractory, locally advanced or metastatic differentiated thyroid cancer: a randomised, double blind, phase 3 trial. Lancet. 2014;384:319-328.

24. Khakoo AY, Kassiotis CM, Tannir N, et al. Heart failure associated with sunitinib malate: a multitargeted receptor tyrosine kinase inhibitor. Cancer. 2008;112:2500-2508.

25. Chu TF, Rupnick MA, Kerkela R, et al. Cardiotoxicity associated with tyrosine kinase inhibitor sunitinib. Lancet. 2007;370:2011-2019.

26. Schwandt A, Wood LS, Rini B, Dreicer R. Management of side effects associated with sunitinib therapy for patients with renal cell carcinoma. Onco Targets Ther. 2009;2:51-61.

27. Autier J, Escudier B, Wechsler J, Spatz A, Robert C. Prospective study of the cutaneous adverse effects of sorafenib, a novel multikinase inhibitor. Arch Dermatol. 2008;144:886-892.

28. Flaherty KT, Brose MS. Sorafenib-related hand-foot skin reaction improves, not worsens, with continued treatment. Clin Cancer Res. 2009; $15: 7749$

29. National Comprehensive Cancer Network. Clinical Practice Guidelines in Oncology. Cancer related fatigue. Available from: http://www.ncen. org/professionals/physician_gls/f_guidelines.asp. Accessed July 24, 2015.

30. Carhill AA, Cabanillas ME, Jimenez C, et al. The noninvestigational use of tyrosine kinase inhibitors in thyroid cancer: establishing a standard for patient safety and monitoring. J Clin Endocrinol Metab. 2013;98:31-42.

31. Najjar YG, Mittal K, Elson P, et al. A 2 weeks on and 1 week off schedule of sunitinib is associated with decreased toxicity in metastatic renal cell carcinoma. Eur J Cancer. 2014;50:1084-1089.

32. Atkinson BJ, Kalra S, Wang X, et al. Outcomes associated with sunitinib alternative schedule compared to traditional schedule: a single-center experience. J Clin Oncol. 2013;31 Suppl 15:e15611.

33. Zimmermann K, Schmittel A, Steiner U, et al. Sunitinib treatment for patients with advanced clear-cell renal-cell carcinoma after progression on sorafenib. Oncology. 2009;76:350-354.

34. Cabanillas ME, Waguespack SG, Bronstein Y, et al. Treatment with tyrosine kinase inhibitors for patients with differentiated thyroid cancer: the M. D. Anderson experience. J Clin Endocrinol Metab. 2010;95: 2588-2595.

35. Illouz F, Braun D, Briet C, Schweizer U, Rodien P. Endocrine sideeffects of anti-cancer drugs: thyroid effects of tyrosine kinase inhibitors. Eur J Endocrinol. 2014;171:R91-R99.

36. Chen L, Shen Y, Luo Q, Yu Y, Lu H, Zhu R. Response to sorafenib at a low dose in patients with radioiodine-refractory pulmonary metastases from papillary thyroid carcinoma. Thyroid. 2011;21:119-124.

37. Frank-Raue K, Ganten M, Kreissl MC, Raue F. Rapid response to sorafenib in metastatic medullary thyroid carcinoma. Exp Clin Endocrinol Diabetes. 2011;119:151-155.

38. Marotta V, Ramundo V, Camera L, et al. Sorafenib in advanced iodinerefractory differentiated thyroid cancer: efficacy, safety and exploratory analysis of role of serum thyroglobulin and FDG-PET. Clin Endocrinol (Oxf). 2013;78:760-767.

39. Carr LL, Mankoff DA, Goulart BH, et al. Phase II study of daily sunitinib in FDG-PET-positive, iodine-refractory differentiated thyroid cancer and metastatic medullary carcinoma of the thyroid with functional imaging correlation. Clin Cancer Res. 2010;16:5260-5268.

40. Ahmed M, Barbachano Y, Riddell A, et al. Analysis of the efficacy and toxicity of sorafenib in thyroid cancer: a phase II study in a UK based population. Eur J Endocrinol. 2011;165:315-322. 


\section{Publish your work in this journal}

OncoTargets and Therapy is an international, peer-reviewed, open access journal focusing on the pathological basis of all cancers, potential targets for therapy and treatment protocols employed to improve the management of cancer patients. The journal also focuses on the impact of management programs and new therapeutic agents and protocols on

patient perspectives such as quality of life, adherence and satisfaction. The manuscript management system is completely online and includes a very quick and fair peer-review system, which is all easy to use. Visit http://www.dovepress.com/testimonials.php to read real quotes from published authors.

Submit your manuscript here: http://www.dovepress.com/oncotargets-and-therapy-journal 